\title{
Development of a marketable rice based herbal porridge suitable for diabetics from Scoparia dulcis
}

\author{
Subhashinie Senadheera, Sagarika Ekanayake* \\ Department of Biochemistry, Faculty of Medical Sciences, University of Sri Jayewardenepura, Sri Lanka; \\ *Corresponding Author: sagarikae@hotmail.com
}

Received July 2013

\begin{abstract}
Consumption of herbal leafy porridges is a reputed dietary remedy among Sri Lankans in treating diabetes. The aim of the study was to develop a rice based herbal porridge [rice: fresh leaves: scraped coconut kernel 13 - 15: 25 - 30: 10 - $13(\mathrm{w} / \mathrm{w} / \mathrm{w})$ ] commercially with Scoparia dulcis leaves which has proven antidiabetic effects and low GI (fresh porridge) which benefits diabetics. Two porridges with the same ingredient ratio were produced with different particle sizes. Porridge produced with $100 \%$ fine particles (extruded rice, leaves and scraped coconut mixed with rice powder) elicited a high GI for normal $(92 \pm 22)$ and diabetic $(97 \pm 20)$ subjects. The second porridge prepared with the minimum amount of extruded rice with other ingredients mixed with boiled and dried intact rice grains had a medium GI (normal $58 \pm 11$, diabetics $61 \pm 11)$. A significant reduction $(p<0.05)$ in the peak blood glucose was observed in diabetic subjects for porridge $2(12.4 \%)$ compared to porridge $1(0.7 \%)$ when compared to glucose. The reason could be the significantly high $(p<$ $0.05)$ amount of larger particles $(>0.5 \mathrm{~mm})(63 \%)$ and minimum amount of extruded fine particles in porridge 2 compared to porridge 1 (1.3\%).
\end{abstract}

Keywords: Herbal Leafy Porridges; Scoparia dulcis; Glycaemic Index; Diabetes

\section{INTRODUCTION}

Diabetes mellitus is one of the five leading causes of deaths in the world. According to WHO in year 2011, 220 million people in the world were suffering from diabetes and this number will increase up to 366 million by 2030 [1]. Consumption of low glycaemic index (GI) foods is a dietary modification that is recommended for diabetic patients by many food and dietetics organizations. GI indicates the change in blood glucose after consumption of starchy foods. Blood glucose spikes following a meal leading to secretion of high insulin is a main factor for the development of obesity which in turn leads to diabetes and other non communicable diseases (NCD) [2]. Low GI foods slow down the rise of postprandial blood glucose level reducing the insulin secretion when compared to high GI foods. Many epidemiological studies have proven the benefits of low GI foods in controlling obesity and other NCDs.

Herbal dietary remedies consumed from ancient times are still widely used [3] to treat hyperglycemic conditions all over the world. Porridge made with herbal extracts (Sinhala-kola kenda) is a unique breakfast food in Sri Lanka which is made using herbal extracts, coconut milk and rice. These are more palatable and fulfilling compared to a water extract of herbs, and are ingested as a meal. Most of the leaves used for the preparation of porridge have scientifically proven hypoglycaemic effects. A previous study with many such porridges proved that most of these porridges [leaves: scraped coconut kernel in 25:15:10 (w/w/w) ratio] have low glycaemic index (GI) values for normal subjects [4]. However, the low GI in porridges was mainly due to the high water content and not due to the hypoglycaemic effects of the leaves as all porridges elicited low GI despite of the proven anti-diabetic effects of the leaves.

A 3-month study with Streptozotocine (STZ) induced diabetic Wistar rats and selected low GI porridges elicited a reduction in weight loss, a decrease in HbA1c and decreased diabetic complications in rats fed with a porridge made of Scoparia dulsic (SD) when compared to a diabetic control and other porridge fed groups [5]. In addition, water extracts, infusions (tea) and tablets of Scoparia dulsic are also used by many diabetic patients all over the world. In today's world 'ready to make foods' are more popular among the urban population. Thus SD porridge which had low GI and elicited better anti-diabetic parameters was selected, to be developed as a commer- 
cial product targeting the diabetic patients. Although the ingredient ratio of freshly prepared and commercially produced porridges is similar, the procedure followed in commercial production included extrusion that may change the texture of the ingredients which may affect the GI. Therefore the objective of this study was to estimate the glycaemic indices of two Scoparia dulsic porridge samples with the same ingredient ratio as in the fresh, low GI porridge but produced commercially, incorporating different rice particle sizes, in normal and in diabetic individuals.

\section{METHODOLOGY}

Preparation of the extruded meal: Tender leaves of SD (15 kg), scraped coconut kernel (10 kg) and red rice [272 6B, Rice Research Institute, Bombuwala, Sri Lanka] (15kg) were mixed and extruded at $110^{\circ} \mathrm{C}-120^{\circ} \mathrm{C}$ using JR 600 Extruder (Instra Por). Extruded meal was dehydrated using a hot air dryer $\left(60^{\circ} \mathrm{C}-75^{\circ} \mathrm{C}, 4-5\right.$ hrs $)$. Dried meal was ground using a Hammer mill (Particle size $100 \%$ pass through $0.5 \mathrm{~mm}$ mesh).

Preparation of porridge 1: Extruded meal prepared as above and rice powder (boiled and dried rice grain powder, $100 \%$ pass through $0.5 \mathrm{~mm}$ mesh) was mixed in 1:1 ratio.

Preparation of porridge 2: Extruded meal prepared as above and boiled and dried intact rice grains were mixed in 1:1 ratio.

Digestible carbohydrate content: Digestible carbohydrate content of porridges 1 and 2 were estimated by modified Holm's method [6]. Porridge 1 and 2 were packed separately (40 g) in air tight packets which contained fresh leaves: rice: scraped coconut kernel in 13 15: 25 - 30: 10 - $13(\mathrm{w} / \mathrm{w} / \mathrm{w})$ ratio.

Estimation of GI and GL: The GI study was designed as a random crossover study. Glycaemic index of porridges 1 and 2 were estimated with 10 healthy individuals (BMI $=18$ - 25, fasting blood glucose level $\leq 110$ $\mathrm{mg} / \mathrm{dl}$, peak blood glucose level after consuming $25 \mathrm{~g}$ of glucose $\leq 180 \mathrm{mg} / \mathrm{dl}$, not under any medication) and with 10 diabetic patients (FBS $>126-300 \mathrm{mg} / \mathrm{dl}$, with no severe complications) [7].

Volunteers were advised to fast for 810 hours and fasting blood glucose measured. Glucose was used as the standard reference food. Volunteers were given $25 \mathrm{~g}$ of glucose in $250 \mathrm{ml}$ of water and blood glucose was measured at 15, 30, 45, 60, 90, 120 minutes after ingestion of glucose. Additional blood samples at 150 and $180 \mathrm{mi}-$ nutes were obtained from diabetic patients.

Incremental area under the curve (IAUC) was calculated using a graph constructed with blood glucose concentration vs. time. The above procedure was repeated twice for every volunteer and the mean IAUC calculated.
Porridges containing $25 \mathrm{~g}$ of digestible carbohydrate (1 packet of each porridge) was given to the volunteers on separate days, after reconstituting the contents in the packet with $500 \mathrm{ml}$ of water and boiling for 5 minutes in a closed pan. IAUC was calculated. The percentage ratio of IAUC for porridge and average IAUC for glucose were taken as GI for each individual. The GI values of each volunteer were averaged to obtain the final GI values.

Glycaemic Load (GL) was estimated by multiplying the Glycaemic Index by the number of net carbohydrates in a given serving (25 $\mathrm{g})$ [8].

Particle size distribution of the porridges: Each porridge (1 and 2) was sieved through $0.5 \mathrm{~mm}, 0.1 \mathrm{~mm}$ and $0.05 \mathrm{~mm}$ sieve set separately and the percentage portion on each sieve and the percentage portion sieved through the final sieve $(0.05 \mathrm{~mm})$ were measured.

Ethical approval: Ethical approval was obtained from the Ethics Review Committee of Faculty of Medical Sciences, University of Sri Jayewardenepura (Approval No.476/09). Written consent was obtained after explaining the procedure to each volunteer.

Statistical Analysis: Data were analyzed using student $t$ test (Microsoft Excel 2007 version).

\section{RESULTS}

Both porridges had comparable digestible carbohydrate contents (68\%) which were similar to the freeze dried fresh porridge. GI values of porridges 1 and 2 for normal and diabetics are stated in Table 1. Porridge 1 elicited high GI in both normal and diabetic individuals. However, porridge 2 elicited medium GI for both normal and diabetic individuals. Porridge 1 had a high GL while porridge 2 had a medium GL for both normal and diabetics.

The glycaemic responses of porridge 1 and porridge 2 in normal and diabetic subjects are given in Figures 1 and 2 respectively. A significant reduction $(\mathrm{p}<0.05)$ in the peak blood glucose was observed in diabetic subjects for porridge 2 (12.4\%) compared to porridge $1(0.7 \%)$ when compared to glucose.

The particle size distribution of porridges 1 and 2 are stated in Table 2. Porridge 2 had a significantly high $(\mathrm{p}<$ $0.05)$ amount of larger particles $(>0.5 \mathrm{~mm})(63 \%)$ compared to porridge $1(1.3 \%)$. The amount of particles smaller than $0.05 \mathrm{~mm}$ in porridge 1 was twice the amount in porridge 2 .

Table 1. GI values of porridges for normal and diabetic individuals.

\begin{tabular}{ccccc}
\hline & \multicolumn{2}{c}{ Porridge 1 } & \multicolumn{2}{c}{ Porridge 2 } \\
\hline & Normal & Diabetic & Normal & Diabetic \\
\hline GI & $92 \pm 22$ & $97 \pm 20$ & $58 \pm 11$ & $61 \pm 11$ \\
GL & $23 \pm 6$ & $24 \pm 5$ & $14 \pm 3$ & $15 \pm 3$ \\
\hline
\end{tabular}

$\mathrm{N}=10$. 


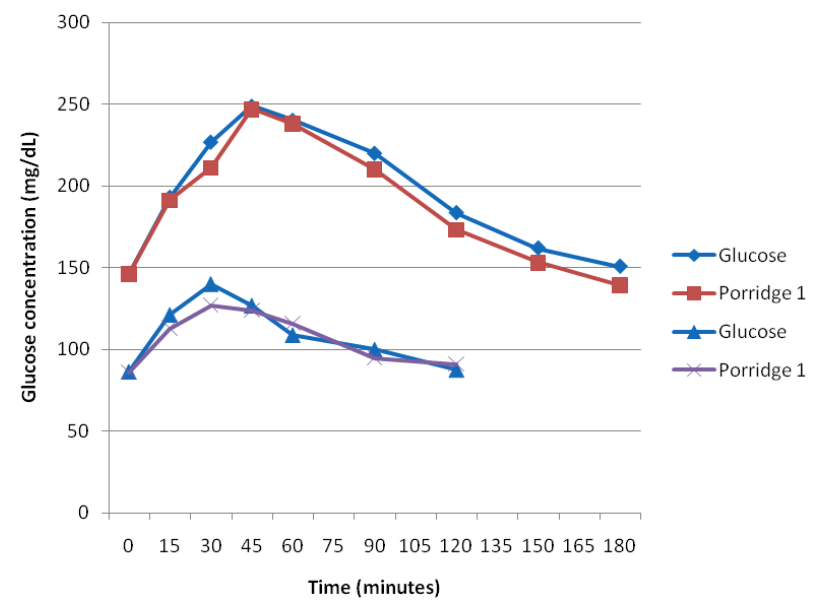

Figure 1. Glycaemic responses of normal and diabetic individuals for porridge 1 (each value represents an average of 10).

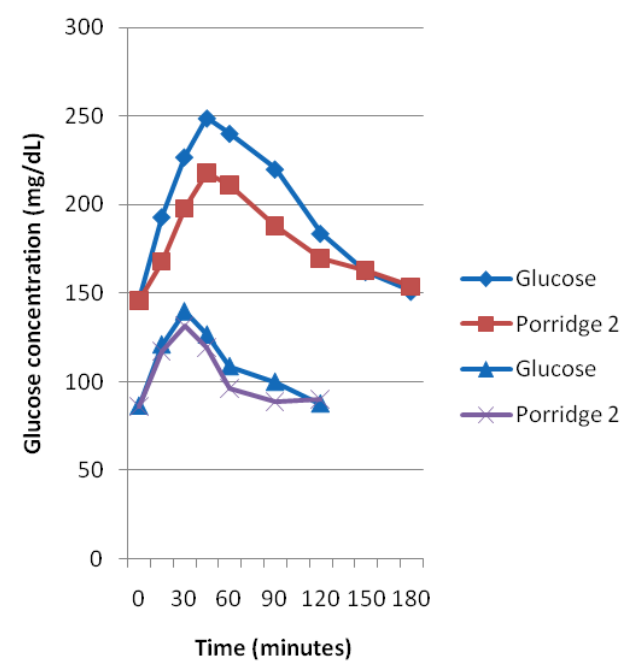

Figure 2. Glycaemic responses of normal and diabetic individuals for porridge 2 (each value represents an average of 10).

Table 2. Particle size distribution of porridges $(n=3)$.

\begin{tabular}{ccc}
\hline & Porridge 1 & Porridge 2 \\
\hline$>\mathbf{0 . 5} \mathbf{~ m m}$ & $1.3 \%$ & $63 \%$ \\
$\mathbf{0 . 5} \mathbf{- 0 . 1} \mathbf{~ m m}$ & $14 \%$ & $0 \%$ \\
$\mathbf{0 . 1} \mathbf{- 0 . 0 5} \mathbf{~ m m}$ & $63.3 \%$ & $24.6 \%$ \\
$<\mathbf{0 . 0 5} \mathbf{~ m m}$ & $20.1 \%$ & $10.2 \%$ \\
\hline
\end{tabular}

\section{DISCUSSION}

Although the ingredients used for the previous study in estimating the GI [4] and for the present study were comparable, in the commercial production process porridge ingredients were extruded. The GI of the commercial porridge 1 with $100 \%$ fine particles (extruded particles + rice powder) elicited a remarkably high GI than the fresh porridge (39 \pm 25 ) used for the previous study. Since the only difference was extrusion which required rice powder and as data reports the effect of particle size of starch based foods on GI [9], minimum amount of rice powder was incorporated in to porridge 2 to retain the palatability and texture, during extrusion procedure. To obtain a similar amount of digestible carbohydrate as in porridge 1 , boiled and dried rice grains were incorporated in to porridge 2 . This elicited a medium GI for both normal and diabetic individuals. This lowering of GI and the reduction in peak blood glucose percentage compared to glucose were more apparent in diabetics than normal individuals indicating the suitability of the porridge 2 on diabetics. This also showed that extrusion procedure gives rise to high glycaemic response.

Since the only difference between the two porridges was the rice particle size, the particle size distribution was analyzed. The medium GI value obtained for porridge 2 with higher particle size distribution, compared to high GI of porridge 1 revealed that particle size of a food is a key factor in controlling the glycaemic response thus the GI. Availability of high surface area for enzyme access might increase the digestibility of foods with fine particles thus increasing the GI. In addition, the high temperatures and pressure the rice grains subjected to during processing may cause gelatinization and cell/starch granule rupture releasing the constituents making these more accessible to enzymatic digestion. These could have contributed for not obtaining the low GI value elicited with freshly made porridge with $100 \%$ intact rice grains. However, since during commercial production addition of rice powder could not be avoided during the extrusion procedure the adjustment made by using minimum rice powder and rice grains have paved the way to reduce the GI to a medium.

\section{CONCLUSION}

Porridge of Scoparia dulcis made incorporating rice grains as in porridge 2 elicits medium GI and medium GL values in normal as well as in diabetics. Therefore porridge produced as porridge 2 is a suitable breakfast food for diabetics which elicits high satiety and hypoglycaemic effects when consumed frequently. This study also proves that porridge with intact rice grains is better for diabetics than powdered porridge.

\section{ACKNOWLEDGEMENTS}

The financial support by ASP/06/PR/2010/12 and PhD/08/2012 grants of University of Sri Jayewardenepura and support provided by Plenty Foods (Pvt) Ltd., Sri Lanka in producing porridge commercially are gratefully acknowledged.

\section{REFERENCES}

[1] World Health Organization, Media Centre (2012) Di- 
abetes: Key facts.

http://www.who.int/mediacentre/factsheets/fs312/en

[2] Temelkova-Kurktschiev, T.S., Koehler, C., Henkel, E., Leonhardt, W., Fuecker, K. and Hanefeld, M. (2000) Postchallenge plasma glucose and glycemic spikes are more strongly associated with atherosclerosis than fasting glucose or HbA1c level. Diabetes Care, 23, 1830-1834. http://dx.doi.org/10.2337/diacare.23.12.1830

[3] Fugh-Beerman, A. (2000) Herb-drug interactions. Lancet, 355, 134-138.

http://dx.doi.org/10.1016/S0140-6736(99)06457-0

[4] Senadheera, S.P.A.S. and Ekanayake, S. (2012) Green leafy porridges: How good are they in controlling glycaemic response? International Journal of Food Science and Nutrition, 64, 169-174.

http://informahealthcare.com/doi/abs/10.3109/09637486. 2012.710895

http://dx.doi.org/10.3109/09637486.2012.710895

[5] Senadheera, S.P.A.S. and Ekanayake, S. (2012) Effect of long term consumption of selected green leafy porridges on liver enzyme profiles of Wistar rats. Annual Sessions, Open University of Sri Lanka, 48.

[6] Holm, J., Drews, A. and Asp, N.G. (1986) A rapid method for the analysis of starch. Starch/Starke, 38, 224-226. http://dx.doi.org/10.1002/star.19860380704

[7] Wolever, T.M.S., Vorster, H.H., Björk, I., Brand-Miller, J., Brighenti, F., Mann, J.I., Ramdath, D.D., Granfeldt, Y., Holt, S., Perry, T.L., Venter, C. and Wu, X. (2003) Determination of the glycaemic index of foods: Interlaboratory study. European Journal of Clinical Nutrition, 57, 475482. http://dx.doi.org/10.1038/sj.ejcn.1601551

[8] Galgani, J., Aguirre, C. and Díaz, E. (2006) Acute effect of meal glycemic index and glycemic load on blood glucose and insulin responses in humans. Nutrition Journal, 5, 22. http://dx.doi.org/10.1186/1475-2891-5-22

[9] Jayasinghe, M.A. and Ekanayake, S. (2013) Effect of different milling methods on glycaemic responses of foods made with finger millet (Eucenea coracana) flour. Ceylon Medical Journal (in press). 